\title{
Letter To The Editor: Comparison of Hypoxanthine and Lactate as Indicators of Hypoxia
}

\author{
OLA DIDRIK SAUGSTAD \\ Department of Pediatrics, Division of Neonatal/Perinatal Medicine, University of California, San Diego, La Jolla, \\ California, USA
}

In a recent paper, "Comparison of Hypoxanthine, Lactate and ECG signs as Indicators of Hypoxia," Tuchschmid et al. (3) determined venous lactate and hypoxanthine levels in adults at an altitude of $6000 \mathrm{~m}$. No alterations in hypoxanthine concentrations were found either during rest $(27 \mathrm{~min})$ or minimal exercise $(4$ min). During rest, the lactate levels were elevated, but did not exceed the normal range. During exercise, a small but significant increase in lactate levels was demonstrated. The authors concluded that "hypoxanthine in isolated, acute hypoxemic hypoxia in young adults is a less sensitive parameter than lactate" (3). Before such a conclusion can be drawn, however, it is important to be aware that hypoxanthine and lactate accumulation do not necessarily describe the same situation, but that different information may be given by these two measurements.

In addition to a series of conditions not associated with hypoxia, elevated lactate levels are found during hypoxia and reflect increased glycolysis to meet the increased energy demands of the hypoxic cell. Thus, even with high lactate level, the energy status of the cell is not necessarily altered. The elevation of hypoxanthine, on the other hand, probably reflects a deterioration of the energy charge. Therefore, there will be a dissociation between lactate and hypoxanthine patterns found, at least in mild hypoxia. An increased lactate level with normal hypoxanthine values thus can represent a compensated energetic stress situation. If the hypoxanthine level is elevated in addition to lactate, there is uncompensated energetic stress tending toward a hypoenergetic state.

In most of our experimental data, the hypoxia was to go deep to discover such a dissociation between these two metabolites ( 1 , 2 ), and there was an excellent correlation between lactate and hypoxanthine. The augmentation of hypoxanthine was so fast that there was no acidosis or cell destruction which could explain at least the very early hypoxanthine elevation, as proposed by Tuchschmid et al. in their conclusion. Other authors have found elevated oxypurine levels at high altitude (4), although the duration was much longer than that reported by Tuchschmid et al. (3). There also was a close correlation between plasma oxypurine (hypoxanthine + xanthine) and oxygen saturation in arterial blood (4) in contrast to the finding of Tuchschmid et al. (3). The data presented by Tuchschmid et al. probably represent compensated energetic stress. This is not surprising if one looks at the very small lactate elevations these authors actually found. It is not surprising either that healthy adults have energy reserves which can compensate 4 min minimal work load, although performed at high altitude. We think care should be exercised in drawing firm conclusions from the data presented by Tuchschmid et al. Only four subjects were tested, and the lactate elevation was only from $1.08 \pm 0.24$ to 1.41 $\pm 0.42 \mathrm{mmoles} /$ liter after the working load was finished. It is obvious that factors other than hypoxia may be responsible for this modest lactate elevation. It has really not been ruled out, as discussed by the authors themselves, that, for instance, hyperventilation, which occurred in these subjects, can explain, at least partly, the moderate lactate elevations.

\section{REFERENCES AND NOTES}

1. Saugstad, O. D., Aasen, A. O., and Hetland, Ø.: Plasma hypoxanthine levels in pigs during acute hypoxia. Eur. Surg. Res., 10: 314 (1978).

2. Thiringer, K., Saugstad, O. D., and Kjellmer, I.: Plasma hypoxanthine in exteriorized, acutely asphyxiated fetal lamb. Pediatr. Res., 14:904, 1980.

3. Tuchschmid, P. E., Boutellier, U., Koller, E. A. and Duc, G. V. Comparison of hypoxanthine, lactate, and ECG signs as indicators of hypoxia. Pediatr. Res., 15: 28 (1981).

4. Yoshino, M. Hayashi, R. Katsumata, Y. Mori, S., and Mitarai, G.: Blood oxypurines and erythrocytes 2,3-diphosphoglycerate levels at high altitude hypoxia. Life Sci., 27: 1265 (1980).

\section{Letter to the Editor: Signs of Mild Hypoxia}

\author{
P. E. TUCHSCHMID AND G. V. DUC
}

Division of Neonatology, Department of Pediatrics, University of Zurich, Zurich, Switzerland

Neonatologists have been looking for a chemical indicator of mild and/or past hypoxia in newborns. Base-excess, standardbicarbonate, and lactate were used. Concentration of hypoxanthine was recently found to be elevated in plasma of hypoxic newborns (1) and in animal-models of hypoxemic (3) and ischemic (4) hypoxia. Our investigation (6), published in the January issue of this journal, compared the sensitivity of this parameter with lactate elevation and ECG-signs of myocardial-hypoxia as indicators of hypoxia. Altitude-induced hypoxemia associated with work in healthy adults was studied. The results show that lactateelevation and ECG signs of hypoxia preceed the elevation of hypoxanthine, which was augmented only in part of the cases where its in vivo breakdown was inhibited by allopurinol. We concluded that in mild hypoxia hypoxanthine is a less sensitive 
parameter than lactate and ECG changes. In his letter to the editor (2), Dr. Saugstad does not accept the conclusions drawn from these results for the following reasons:

1. The observed lactate elevation during work at high altitude could be caused by hyperventilation and not by hypoxia.

Work-induced hyperventilation was found in only one of nine volunteers exposed to high altitude. This case is analysed separately as shown in Table 1 of our paper (6). Hyperventilation as assessed by $\mathrm{P}_{\mathrm{A}} \mathrm{CO}_{2}$ can be ruled out as the cause of lactate elevation during work.

2. The observed lactate elevation could be caused by a relative augmentation of the energy demand without true hypoxia ("compensated energetic stress") (2).

If lactate is accepted as a marker of unbalance between glycolysis and citric acid cycle, assuming unchanged $\mathrm{pH}$ and $\mathrm{P}_{\mathrm{A}} \mathrm{CO}_{2}$, then any elevation of lactate measured a few minutes after the onset of work is an expression of hypoxia at least in part of the organism (5). ECG data of our experiments (6) show that in fact the myocardium was hypoxic.

Whether we call this situation "compensated energetic stress" (2) or "mild hypoxia" (6) does not weaken the fact that we were able to produce experimental hypoxemic conditions with lactate elevation, hypoxic ECG signs but without changes in the hypoxanthine concentration in plasma. Our data do not contradict the findings (1) that hypoxanthine is elevated in hypoxia, but they indicate that absence of hypoxanthine elevation does not rule out mild hypoxia. The short half-life of hypoxanthine in vivo (4) relativates its value as an indicator of past hypoxia.

\section{REFERENCES AND NOTES}

1. Saugstad, O. D.: Hypoxanthine as a measurement of hypoxia. Pediatr. Res., 9 158 (1975)

2. Saugstad, O. D.: Letter to the editor: comparison of hypoxanthine and lactate as indicators of hypoxia. Pediatr. Res. 15: 1140 (1981)

3. Saugstad, O. D.. Aasen, A. O., and Hetland, Ø.: Plasma hypoxanthine levels in pigs during acute hypoxemia. Eur. Surg. Res., 10: 314 (1978)

4. Saugstad, O. D., Kroese, A., Myhre, H. O., and Andersen, R.: Alteration of plasma hypoxanthine concentration during ischemia in the forelimb of the pig. Scand. J. Clin. Lab. Invest., 37: 517 (1977).

5. Takano, N.: Role of hypocapnia in the blood lactate accumulation during acute hypoxia. Respir. Physiol., 4: 32 (1968).

6. Tuchschmid, P. E., Boutellier, U., Koller, E. A., and Duc, G. V.: Comparison of hypoxanthine, lactate, and ECG signs as indicators of hypoxia. Pediatr. Res. 15: 28 (1981). 